\title{
Penile Prosthesis in the Age of the Blue Pill
}

\section{R. Andrianne}

Urology Department, Cetism (Centre de Traitement de l'Insuffisance Sexuelle Masculine), Liège University Hospital, Liège, Belgium.

\section{Introduction}

In the event of proven erectile dysfunction (ED), specific treatment strategies are available in the following order: phosphodiesterase type 5 inhibitors (Cialis, Levitra, Viagra), intracavernous injections of vasoactive drugs and implanting a penile prosthesis. According to clinical experience, with all causes taken into consideration, $25 \%$ of patients who suffer from ED do not respond to medical treatment. The penile implant is a highly effective alternative treatment where oral or injected drug therapy has proven unsuccessful or in cases where other medical treatments are contraindicated (1). According to our experience, the number of implant procedures performed increases by $15 \%$ each year and 35 prostheses will be implanted in 2009. Unfortunately, the penile implant remains a marginal therapy in Belgium despite being highly effective, having a very low complications rate and a satisfaction rating over $90 \%$. Around 30 to 45,000 implant procedures are performed each year throughout the world with ten times more surgeries in the US than in Europe. In 2008, only 140 penile implants were performed in Belgium. There are an estimated 30 urologists who perform this procedure and only two among this number perform more than 25 implant procedures per year. Therefore the majority of surgeons performing the procedure have less experience (three implants per year on average). This is not an ideal situation. As is the case in many European countries, it would appear that the low number of penile implants is a result of a lack of training and a shortage of information in the field, the cost of the implant, perioperative failings or complications in the case of insufficient experience. The medical legal risk is probably over-estimated but it does exist. The vast majority of implant models are inflatable. This advance, alongside the highly-informed support of doctors that are sensitive to ED patients and their partners' quality of life, should make the process of deciding to get an implant more straight-forward.

\section{When is it time to consider a penile implant ?}

In the beginning the arrival of Sildenafil in 1998 reduced the number of implant procedures performed. The number of patients requesting rehabilitation quickly increased and the number of implant procedures performed rose swiftly in the USA. Organic erectile dysfunction that has not responded to medical treatment in a motivated patient and partner is the best indication for a penile implant. Contraindication against other medical treatments is also a good reason to opt for a penile implant (treatment with nitroderivatives, etc.). If the erectile situation is very detrimental, then the rehabilitation request is often significant. Ideally, tests (vascular and/or neurological) should show an irreversible alteration of the erection processes. The main causes of ED are vascular, linked to diabetes, following pelvic surgery (radical prostatectomy) and neurological. Peyronie's disease is also a cause of erectile dysfunction which resists medical treatment. Here surgery can correct the abnormal curvature (tunica albuginea graft or manual shaping on the implant) and ED at the same time.

In patients suffering ED, physicians regularly observe a reduction in penis size which is exacerbated in certain situations, such as Peyronie's disease, after radical prostatectomy, after low-flow priapism and in paraplegic patients. We believe that the indication for surgery should not be proposed too late as the aesthetic and functional results depend on the size of the implant used. Certain patients will not be selected to undergo the implant procedure. These are basically patients who have not been informed on alternative treatments, patients with psychological issues who have not received suitable psychological treatment, patients with general surgical risks or with severe cavernous fibrosis. Certain badlyinformed patients do not have realistic expectations of the benefits of a penile implant and in such cases, as is our usual practice and after providing information, we endeavour to have them anonymously meet with patients who have already been operated on. Symptoms of intravesical obstruction or infections in the penis (balanitis, phimosis) will have to be treated prior to implantation. The patient is required to sign an informed consent, as getting a penis implant is a procedure that is influenced by considerable psycho-emotional expectations and therefore, has high medical legal risks. The patient must be aware that he will no longer be able to achieve erections naturally, though they will be extremely fulfilling. He should also be informed that the implantation procedure is irreversible and that re-operation may be required , as is inherent to any prosthetic procedure. Repeat 


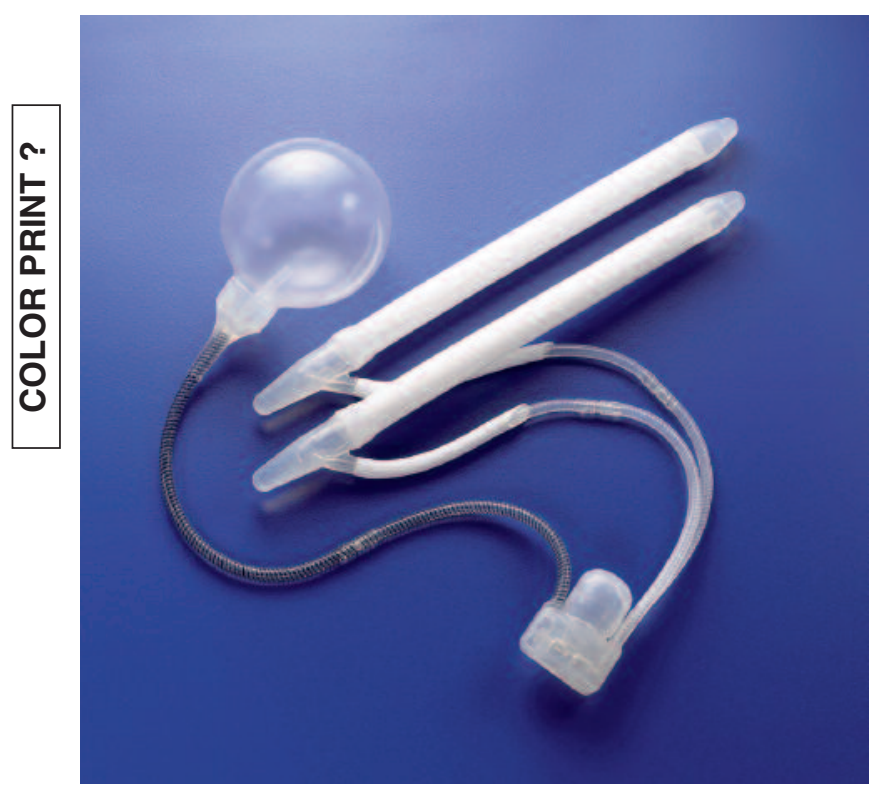

Fig. 1

Three piece CX AMS 700 penile prosthesis

surgery and replacement of the prosthetic element is most often linked to a mechanical problem particularly with hydraulic implants (leaks, cylinder failure, etc.) and is sometimes required by rare pain phenomena $(6 \%)$, infection or erosion (5 to $6 \%$ ) and sometimes a technical error in the choice of implant (5 to 6\%). The infection rate, which is the most dangerous complication, drops with the surgeon and his team's level of experience. Infection may appear soon after the operation in the case of particularly virulent germs or later caused by germs with low virulence levels. In the event of infection, the most commonly encountered germ is Staphylococcus epidermidis. Infection requires a course of treatment with antibiotics and the material must be totally removed . In most cases a salvage procedure by replacing the old implant with a new one after washing in antibiotic solution is possible. This procedure is effective in $80 \%$ of cases in the absence of significant erosion.

Soaking new products in antibiotics enables us to reduce infection rates (excluding diabetics) to below $2 \%$. Mechanical failure rates are currently dropping off due to improvements in the material being implanted. Implanting a penile prosthesis is a straight-forward surgical procedure for a surgeon who has received specific training and has regular practice.

What a patient, interested in a penile implant, needs to know :

- the other options available for treating ED,

- what the implant can do for him and the changes to his body-scheme,

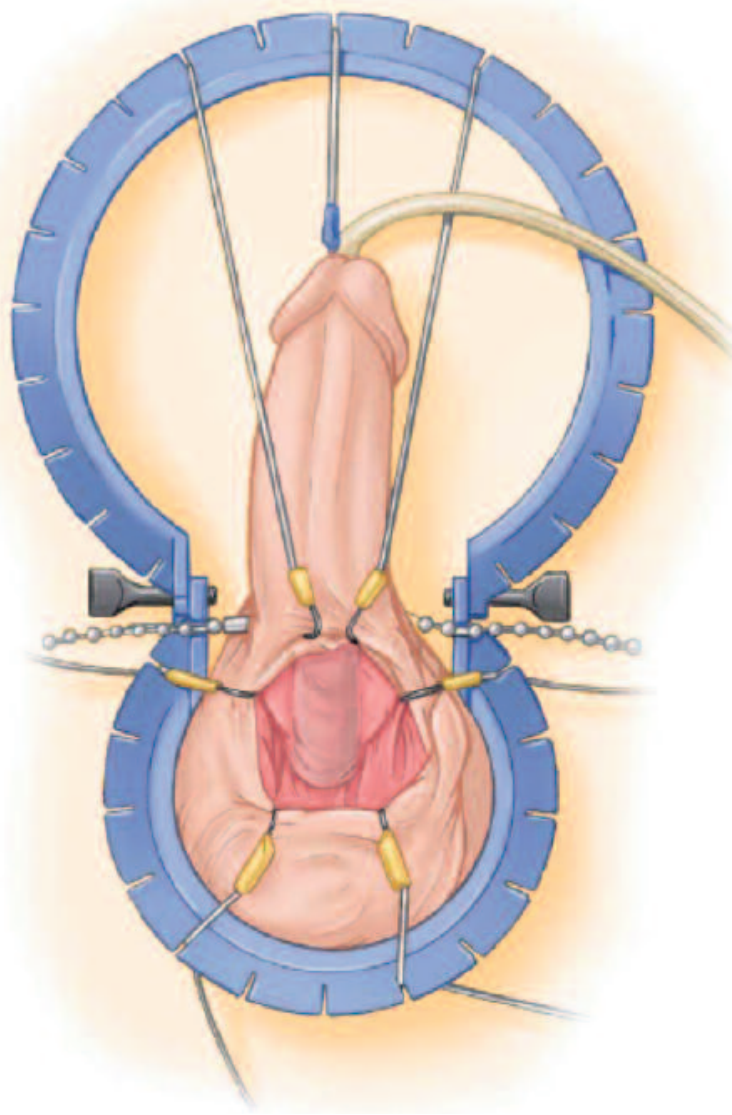

Fig. 2

Penoscrotal approch for penile prosthesis implantation

- the functional and cosmetic design,

- meet with other patients who have been operated on,

- get explanations regarding possible complications : the worst-case scenario, the major complications and how often they occur,

- the possibility of revision surgery.

\section{The different types of implants}

There are basically two types of penile implant :

- semi-rigid implants

- inflatable implants

Inflatable implants are composed of two or three pieces. The three-piece model provides the best flaccidity. New inflatable implant models enable the implant to be lengthened and the diameter to be increased with excellent flaccidity comfort levels. For several years now treatments on the surface of the implant (Minocycline and Rifampicin coating) have reduced the risk of infection by half.

Two manufacturers share the inflatable implant market : AMS INC. USA and MENTOR CORP. USA. 


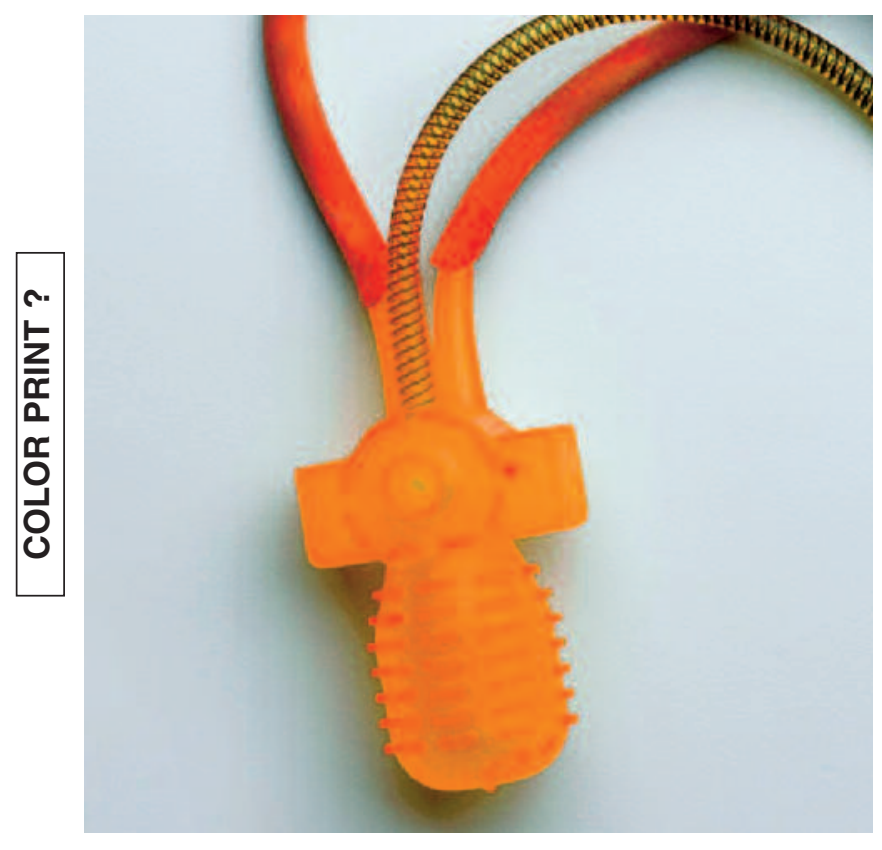

Fig. 3

New scrotal Tactile Pump from AMS-Antibiotic inbibition

\section{Which implant for which patient?}

The implant model that is chosen will depend on the patient's background and dexterity, on whether the patient suffers from Peyronie's disease or fibrosis in the cavernous bodies. The length of the cylinders and the size of the reservoir will be chosen during the operation after measuring the space available in the cavernous bodies. If the penis retains good elasticity, then it will have an optimal cosmetic look when erect.

\section{Which surgical approach?}

The pre-pubic area or the penoscrotal junction are the most commonly used approaches for making a small incision. All the prosthetic components (cylinders, scrotal pump and the abdominal reservoir in the case of a 3-piece implant) can all be implanted through this single incision. If the patient has undergone previous pelvic surgery, a low iliac fossa approach is useful for inserting the balloon containing the saline solution. The operation, which is quick in the hands of highly-trained staff, usually lasts just over one hour. Spinal anaesthesia is the best option and allows to obtain an optimal sizing of the length of the prosthesis to be implanted.

\section{What is the cost for the patient?}

Patients of this surgical procedure are entitled to a very low refund on the procedure itself (K120=150 Euros), on the other hand in Belgium, the material has been subject to an $80 \%$ refund since 1992 with the condition that the ED has a proven vascular and/or neurological cause. This means that this procedure would cost the patient 1000 Euros for a shared room. Patients stay in hospital for two days following surgery.

\section{The importance of well-informed consent}

There must be full well-informed consent from the patient and ideally from his partner also for the surgery. The patient must be informed of the types of implant
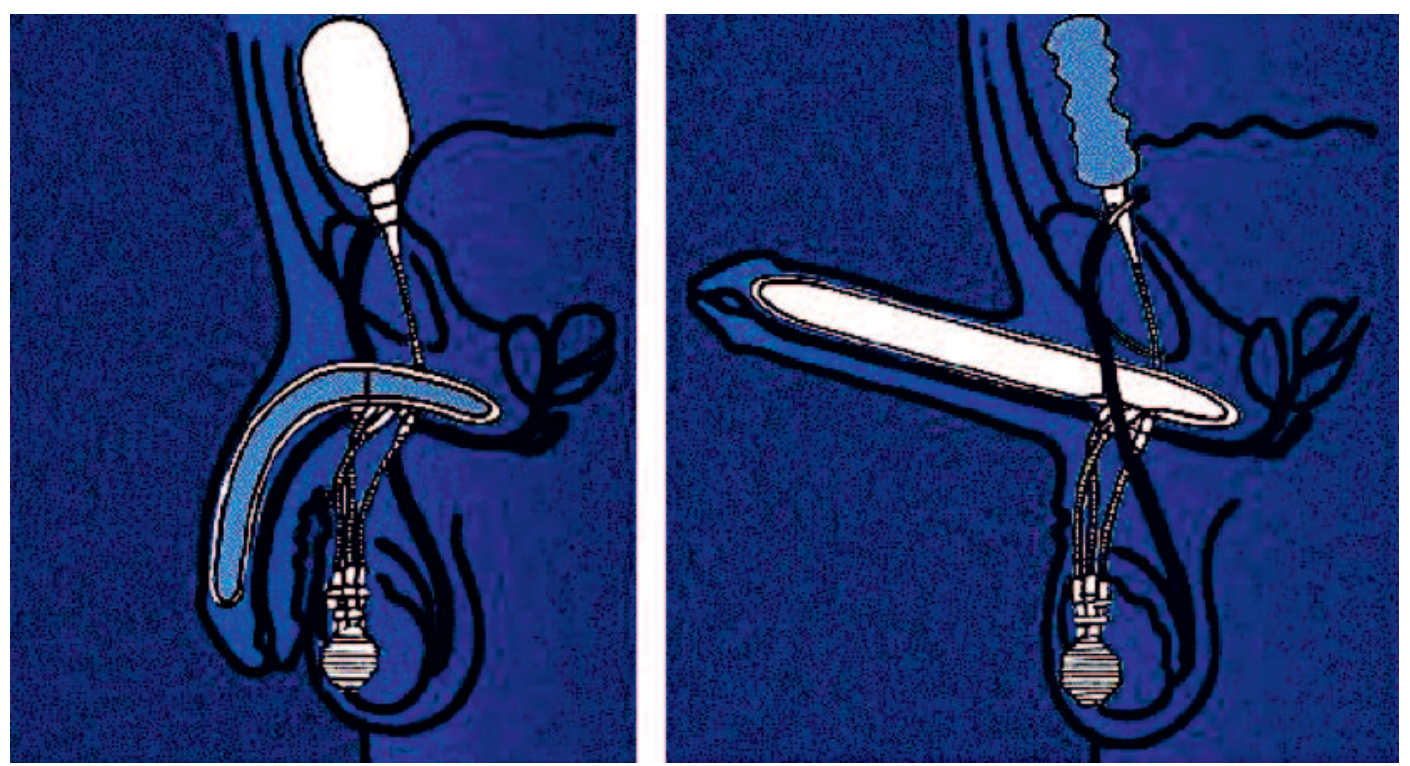

Fig. 4

Penile prosthesis in flaccid state (left) and erection state (right) 
available, the risk of infection, the risk of mechanical malfunction and the need for revision surgery. He must be aware that the erection will no longer be fully natural and that the size which normally doubles in length when there is no erectile dysfunction will be that of the implant without tissue tension. When there is no sexual stimulation the glans will not present an erection and phosphodiesterase type 5 inhibitors are sometimes needed to improve the erection in the glans during sexual relations. The patient must be fully aware that the procedure is irreversible as cavernous tissue is destroyed when the cylinders are inserted. It is most often the case that penile sensitivity, libido and ejaculatory orgasm are improved after penile implant surgery whose sole aim is erectile restoration. A preoperative urine cytology test is essential as is a glycated haemoglobin test for diabetic patients in order to optimise healing and reduce the risk of infection.

\section{Satisfaction with the implant}

According to our own experience and that of several authors (2-4), a penile implant is the most effective treatment of ED and more than $90 \%$ of patients are satisfied on a lasting basis. This in comparison with $50 \%$ for Sildenafil, and $40 \%$ for intracavernous injections (2). Our statistics for 392 procedures from 1983 to 2009 show 109 replacements (89 patients (22.7\%) due to wear or mechanical problems -20 patients $(5.1 \%)$ due to problems with infection)

Only five patients who received implants during this period have no implant today. Our patients' satisfaction is significant : $86 \%$ would recommend the procedure to someone else, $85 \%$ would accept an analogous procedure if necessary. This can be compared to published American statistics (5) 93\% of partners are happy with the operation and $89 \%$ feel pleasure when using this device during sexual relations (6).

\section{Conclusion}

\section{Benefits of penile implants}

- Offers a permanent, long-term solution to ED

- Provides the ability to have an erection at any time

- Allows for greater spontaneity when initiating sexual relations

- Enables the patient to maintain an erection for as along as he desires
- Eliminates the need for costly medication with side effects or contraindications

- Does not interfere with ejaculation or orgasm

\section{Disadvantages of penile implants}

- Causes the permanent destruction of cavernous tissue (which was incapable of achieving an erection)

- If an infection occurs, the implant must be removed

- Requires surgery and a short healing period

- In rare cases may cause a shortening of the penis when erect

- In the case of the inflatable models, the implant could fail

A penile implant may be advisable in cases where oral or intracavernous drug treatments have failed among motivated and well-informed patients. Despite the idea of a last resort treatment, the models available nowadays are very reliable and the satisfaction rate at the medium and long term is very high after a procedure with a low complication rate. Ideally, penile implants should be inserted in a specialised center.

\section{References}

1. Moncada I., Juan I. Current role of penile implants for erectile dysfunction. J Impot Res, 2004, 16 : 57-59.

2. Rajpurkar A., Dhabuwala C. B. Comparison of satisfaction rates and erectile function in patients treated with sildenafil, intracavernous prostaglandin E1 and penile implant surgery for erectile dysfunction in urology practice. J Urol, 2003, 170 : 159-163.

3. Carson C. C., Mulcahy J. J., Govier F. E. Efficacy, safety and patient satisfaction outcomes of the AMS 700CX inflatable penile prosthesis. J Urol, 2000, 163 : 476-480.

4. Agrawal V., Ralph D. An audit of implanted penile prosthesis in the U.K. BJU International, 2006, 98 : 393-395.

5. Kempeneers P., Andrianne R., Mormont C. Penile prosthesis, sexual satisfaction and representation of male erotic value. Sexual and relationship Therapy, 2004, $19: 379-392$.

6. Levine L. A., Estrada C. R., Morgentaler A. Mechanical reliability and safety of inflatable penile prosthesis : results of a 2 center study. J Urol, 2001, 166 : 932-937.

\section{R. Andrianne}

Urology Department

Cetism (Centre de Traitement de l'Insuffisance Sexuelle Masculine)

Liège University Hospital

Sart Tilman B30

4031 Liège, Belgium

E-mail : robert.andrianne@chu.ulg.ac.be 